\title{
Nonrelational processing of a sequential duration discrimination by pigeons
}

\author{
PHILIPP J. KRAEMER \\ Purdue University, West Lafayette, Indiana
}

\begin{abstract}
Pigeons were trained on a conditional discrimination involving signal duration. Red and green keylights were presented in succession for different durations. The pigeons were then allowed to choose between the two colors, and responding to the color that had appeared for either the shorter or the longer duration was reinforced. Following acquisition, probe trials were presented in which only one color appeared. Performance on these trials indicated that the pigeons had not learned to process the two duration signals in a relational manner, but instead based responding exclusively on the color with longer duration. Implications for the study of representation in animals are considered.
\end{abstract}

This experiment represents the outcome of a failed project. The initial objective was to study short-term retention in pigeons trained to discriminate relative stimulus duration. This objective was aborted, however, as a result of the outcome of the experiment to be described. Yet although the primary question was abandoned, the results of this experiment do offer some important insights.

The pigeon's ability to accurately time events is well documented (Church, 1984; Killeen \& Fetterman, 1988). More complex expression of this ability has been demonstrated by pigeons trained on conditional discriminations that require duration comparisons. Reinforced responding in these situations is conditional on either the absolute duration of the test stimuli (Spetch \& Wilkie, 1983) or the relative duration of two or more stimuli (Fetterman \& Dreyfus, 1987). In a duration-comparison procedure, subjects are presented with two or more successive signals, each presented for a different duration. Reinforced responding is contingent on the relative duration of the signals and follows a specific response rule, such as "respond to the left key if signal $A$ was longer than signal B, or to the right key if B was longer than A" (Fetterman \& Dreyfus, 1986, 1987). Rather than make a spatial response, pigeons can also be trained to respond selectively to properties of the signals themselves. For example, Dreyfus, Fetterman, Smith, and Stubbs (1988) trained pigeons on a task in which red and green keylights appeared in succession, each for a different duration. The two colors were then presented simultaneously on the sidekeys, with reinforced responding to the color that had appeared for the longer duration. The pigeons were able to reach high levels of accuracy on this task, even though the absolute durations of the two stimuli varied over trials.

The present research was supported by a grant from the National Institute of Mental Health (1R01 MH44082-01) to Philipp J. Kraemer, who is now in the Department of Psychology at the University of Kentucky, Lexington, KY 40506.
The experiment to be reported here was nominally designed as a duration-comparison procedure, but it was discovered that the pigeons were responding without relying on relative duration. The procedure was similar to that followed by Dreyfus et al. (1988), with the following exceptions: Only two values of duration were used across trials ( 2 and $8 \mathrm{sec}$ ); the pigeons were trained to choose the color of the signal that appeared for either the shorter or the longer duration; and the serial order of the red and green stimuli alternated over trials, which made serial position an irrelevant cue. Each trial consisted of a successive presentation of red followed by green or of green followed by red; one color appeared for $2 \mathrm{sec}$ and the other for $8 \mathrm{sec}$. This was followed immediately by a choice between red and green. Two pigeons were reinforced for choosing the color presented for $2 \mathrm{sec}$, and 2 other pigeons were reinforced for choosing the color presented for $8 \mathrm{sec}$. The primary goal was to examine performance at various delays imposed between termination of the duration cues and presentation of the choice stimuli. Prior to this test, however, the preliminary experiment reported here was conducted. It was designed to determine whether or not the pigeons were actually using the relative durations of the red and green signals to guide performance. It involved presentation of probe trials in which only a single color cue appeared, for either 2 or $8 \mathrm{sec}$. The highly systematic performance on the singleduration problem trials led to the conclusion that relative duration was not the basis on which the pigeons were responding.

\section{METHOD}

Subjects

Four adult White Carneaux pigeons served as subjects. Each pigeon had prior experience with an identity matching-to-sample problem, involving pattern stimuli. They were housed in individual stainless steel hanging cages, located in a vivarium maintained on a 12:12-h light:dark cycle. All pigeons were maintained at $85 \%$ ad-lib weights throughout the experiment, and had free access to water. Daily food rations were 
provided after each test session. The pigeons were tested 6 days each week.

\section{Apparatus}

A standard three-key test chamber for pigeons was used for training and testing. Three pecking keys were horizontally arranged on the front wall of the chamber. Each pecking key could be fully illuminated with red or green light. Reinforcement consisted of mixed grain presented from a food well located directly below the center pecking key. The only illumination was that provided by the pecking keys. All trial-event programming and response recording were controlled by a Commodore 64 computer and a locally constructed interface.

\section{Procedure}

All the pigeons were experienced at responding to pecking keys, but they were given an additional two sessions of pretraining, which consisted of reinforced responding to red and green sidekeys. Each trial of acquisition began with the sequential illumination of the center key, red followed by green or green followed by red. Each color appeared for a different duration, either 2 or $8 \mathrm{sec}$. Order of color presentation and duration were counterbalanced across trials. Immediately after termination of the second color signal, red and green appeared on the sidekeys, with the spatial arrangement of the colors balanced across trials. Two birds were assigned to the short correct condition, in which reinforced responses were those directed toward the color that had appeared for $2 \mathrm{sec}$. The two birds in the long correct condition were reinforced for choosing the color that appeared for $8 \mathrm{sec}$. The eight possible trial types ( 2 colors $\times 2$ durations $\times 2$ spatial locations) were presented eight times within each 64-trial session. The order in which the trial types appeared was randomized within each session for each subject. A 15-sec intertrial interval began after either termination of reinforcement on correct trials or an incorrect response. Training continued until a criterion was achieved of $85 \%$ correct or better on five consecutive sessions. The test sessions began the day after criterion was reached.

Test sessions were identical to training sessions, except that eight additional probe trials were embedded within each session. The probe trials consisted of single-color presentations, in which either red or green appeared alone for 2 or $8 \mathrm{sec}$ on the center key. Responses on probe trials were not reinforced. Each of the eight possible probe trials ( 2 colors $\times 2$ durations $\times 2$ locations of the choice stimuli) was presented once within a session. Within each 72 -trial session, the trial types were randomly arranged for each subject, with the restriction that one probe trial appear within each 9-trial block. Testing continued for 12 sessions.

\section{RESULTS}

Choice responses on probe trials were classified as correct according to the original contingencies. For example, if a pigeon had been trained to choose the color that had appeared for the shorter duration $(2 \mathrm{sec})$, then on a singleduration probe trial a response to a color that appeared for $2 \mathrm{sec}$ was correct, and a response to a color that appeared for $8 \mathrm{sec}$ was incorrect. The performance of each subject and the mean group performance appear in Figure 1. Accuracy is plotted as percent correct for the three trial types: standard two-duration, long probe, and short probe. It can be seen that performance was uniform across subjects. Accuracy on compound durations was equivalent to that on long-duration probe trials, and it was substantially higher on both of these trial types than on short probe trials. The percent correct scores were evaluated with a $2 \times 3 \times 3$ mixed factorial analysis of variance. The between-groups factor was the correct duration $(2$ or $8 \mathrm{sec})$; the two within-group factors were trial type (two-duration, short probe, or long probe) and trial blocks (the 12 trials were divided into three blocks of four

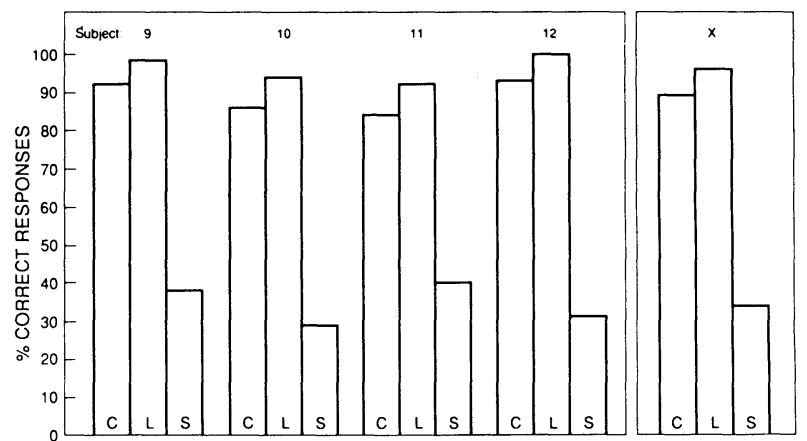

Figure 1. Mean correct responses for the three trial types $(C=$ standard two-duration trial, $L=$ long-duration probe trial, and $S=$ short-duration probe trial).

trials each). The only significant effect was that due to trial type $[F(2,35)=183.9, p<.001]$. Individual mean comparisons revealed that accuracy was equivalent on the standard two-duration trials and long probe trials. Also, accuracy was significantly higher on both of these trial types than on short probe trials (Fisher, $\alpha=.01$; Keppel, 1982). Analysis of the mean number of peck responses to the center key indicated that the pigeons responded significantly more often to 8-sec colors $(M=$ 15.3) than to 2 -sec colors $(M=3.3)[t(3)=9.16$, $p<.01]$, but the rate of responding was equal for the two durations.

\section{DISCUSSION}

The unambiguous results lead to a simple conclusion: The pigeons in this experiment were not processing relative durations of the red and green signals, but were instead responding on the basis of the color of the long-duration signal alone (the color that appeared for $8 \mathrm{sec}$ ). The equally high levels of accuracy on long probe trials and standard twoduration trials indicates that responding to long-duration signals alone was the same as it would have been had a short-duration signal also been presented. Performance on short-duration probe trials was decidedly different. The persistent below chance level of accuracy on these trials indicates that responding occurred as if a long-duration signal alone had been presented. This suggests that, on compound trials, all 4 pigeons ignored the 2-sec signal and based their responding exclusively on the color of the 8-sec signal. It seems that for the 2 birds trained to choose the longer duration color, the strategy was to employ an identity-matching rule-"choose the color of the 8-sec signal." The other 2 birds, which were trained to choose the color that appeared for $2 \mathrm{sec}$, behaved as if they were using an oddity rule-" choose the alternative to the color that appeared for $8 \mathrm{sec}$." Both rules could then have been extended to probe trials by simply applying the identity or oddity rule to the single color that was presented, regardless of its duration. Thus, although nominally intended as a relative duration comparison task, the pigeons seem to have adopted an algorithm that allowed them to solve the task without necessarily processing relative duration. On what basis the pigeons selected the long-duration color as the critical cue for implementing the identity or oddity rule is unclear. It could have involved some threshold of either duration or number of keypecks, but the available data do not provide evidence on this matter.

Dreyfus et al. (1988) also considered the possibility that the pigeons in their experiment may have used absolute duration of one of the signals to guide performance, rather than relative duration. On the basis of statistical analyses, they concluded that although absolute duration may have been used on some trials, relational information was also used. There are two possible ways to approach the different conclusions reached in the two studies. One possibility is that had Dreyfus et al. used a single- 
duration probe procedure, their conclusion may have been different. Perhaps they too would have discovered evidence that their pigeons were utilizing a strategy other than relational processing, which may have been masked in their analysis of performance on compound-duration trials. A second and more likely possibility is that the procedure used by Dreyfus et al. did evoke relational processing, whereas the procedure used in the present experiment did not. Dreyfus et al. employed a wide variety of durations across trials; the present experiment contained only two durations. It may be that the use of an extended set of durations is a necessary condition for the establishment of relational processing, and that using a restricted range of durations promotes nonrelational processing. Another procedural difference is that in the present experiment the serial order of the red and green signals varied across trials, whereas Dreyfus et al. always presented red followed by green. Thus, serial position was an irrelevant cue in the present experiment, but it was redundant with color in Dreyfus et al. Whether or not this factor could have promoted or discouraged relational processing is unclear.

The contribution of this study is not to show that pigeons do not or cannot process signal durations in a relational manner, nor to challenge the results and interpretations of Dreyfus et al. (1988). Rather, the major points are that arranging stimulus events so as to allow for relational processing does not guarantee that such processing actually occurs, and, more importantly, that a determination as to whether or not relational processing is being used is itself a challenge. The latter involves the old problem of distinguishing nominal and functional stimuli (Underwood, Ham, \& Ekstrand, 1962) with a new twist, which is the need to specify the nature of the algorithms used by animals performing complex tasks. Although often ignored, the determination of algorithms is an essential aspect of the study of representation in animals. In many situations, specification of the functional stimulus, through data-based interference, is sufficient to expose the algorithm, but in some situations it is not. Then it becomes imperative to specify the algorithm first, before considering the question of representational content. For example, the present task could be solved by making a relational judgment concerning the durations of the red and green signals. The subject could then represent the correct choice alternative by its sensorial featuresthat is, color. Alternatively, an algorithm could be used in which the 2 -sec signal is ignored, and only the 8-sec signal is used to direct responding, according to either an identity or an oddity rule. This algorithm could be implemented without invoking a relational judgment concerning the durations of the two signals, which seems to be what occurred in this experiment. Note that the representational content would be the same for both algorithms: the color of the correct alternative.

The present study illustrates that the functional algorithm cannot be assumed from the structure of the task. Additional tests designed explicitly to reveal the algorithm may be necessary. The effort required by such an endeavor seems justified by the implications it may have for the analysis of additional questions about representational processes. As an example, consider a simple conditional discrimination problem, in which Response A is correct following a tone, and Response B is correct following a light. This problem could be used putatively to study and compare short-term storage of auditory and visual memories, by imposing delays between offset of the conditional cue and presentation of the response stimuli. The difficulty is that before any potentially interesting differences in retention for the two modalities could be interpreted, it would be necessary to demonstrate, first, that the subject was actually solving the task by using auditory and visual information. It is entirely possible that in this situation the algorithm could entail a light as opposed to a no light discrimination, rather than tone versus light (cf. Herman \& Forestall, 1985; Kraemer \& Roberts, 1984; Wallace, Steinert, Scobie, \& Spear, 1980). This is a problem germane to many of the issues currently being investigated in animal cognition. As the study of representation in animals progresses, it would seem prudent to deny our intuitions as to how animals solve complex tasks that we design for them. Instead, we should turn to appropriate manipulations and test procedures intended to uncover the algorithms they actually use, before moving on to more interesting issues.

\section{REFERENCES}

Church, R. M. (1984). Properties of the internal clock. In J. Gibbon \& L. Allan (Eds.), Annals of the New York Academy of Sciences: Vol. 423. Timing and time perception (pp. 566-582). New York: New York Academy of Sciences.

Dreyfus, L. R., Fetterman, J. G., Smith, L. D., \& Stubbs, D. A. (1988). Discrimination of temporal relations by pigeons. Journal of Experimental Psychology: Animal Behavior Processes, 14, 349-367.

Fetterman, J. G., \& Dreyfus, L. R. (1986). Pair comparison of duration. Behavioral Processes, 12, 111-123.

Fetterman, J. G., \& Dreyfus, L. R. (1987). Duration comparison and the perception of time. In M. L. Commons, J. E. Mazur, J. A. Navin, \& H. Rachlin (Eds.), Quantitative analysis of behavior: Vol. 5. Reinforcer value - the effect of delay and intervening events (pp. 327). Hillsdale, NJ: Erlbaum.

Herman, L. M., \& Forestell, P. H. (1985). Short-term memory in pigeons: Modality-specific or code-specific effects? Animal Learning \& Behavior, 13, 463-465.

Killeen, P. R., \& Fetterman, J. G. (1988). A behavioral theory of timing. Psychological Review, 95, 274-295.

Kraemer, P. J., \& RoberTs, W. A. (1984). Short-term memory for visual and auditory stimuli in pigeons. Animal Learning \& Behavior, 12, 275-284.

SPETCH, M. L., \& WilkIE, D. M. (1983). Subjective shortening: A model of pigeons' memory for event duration. Journal of Experimental Psychology: Animal Behavior Processes, 9, 14-30.

Underwood, B. J., HAM, M., \& EKstrand, B. R. (1962). Cue selection in paired associate learning. Journal of Experimental Psychology, 64, 405-409.

Wallace, J., Steinert, P. A., Scobie, S. R., \& Spear, N. E. (1980). Stimulus modality and short-term memory in rats. Animal Learning \& Behavior, 8, 10-16.

(Manuscript received July 12, 1989.) 